\title{
Electrical Stimulation for the Treatment of Epilepsy
}

\author{
Paul Boon, Robrecht Raedt, Veerle de Herdt, Tine Wyckhuys, and Kristl Vonck \\ Reference Center for Refractory Epilepsy and Laboratory for Clinical and Experimental Neurophysiology (LCEN), Department \\ of Neurology, Ghent University Hospital, Ghent, Belgium
}

\begin{abstract}
Summary: Despite the advent of new pharmacological treatments and the high success rate of many surgical treatments for epilepsy, a substantial number of patients either do not become seizure-free or they experience major adverse events (or both). Neurostimulation-based treatments have gained considerable interest in the last decade. Vagus nerve stimulation (VNS) is an alternative treatment for patients with medically refractory epilepsy, who are unsuitable candidates for conventional epilepsy surgery, or who have had such surgery without optimal outcome. Although responder identification studies are lacking, long-term VNS studies show response rates between $40 \%$ and $50 \%$ and long-term seizure freedom in $5 \%$ to $10 \%$ of patients. Surgical complications and perioperative morbidity are low. Research into the mechanism of action of VNS has revealed a crucial role for the thalamus and cortical areas that are important in the epileptogenic pro-
\end{abstract}

cess. Acute deep brain stimulation (DBS) in various thalamic nuclei and medial temporal lobe structures has recently been shown to be efficacious in small pilot studies. There is little evidence-based information on rational targets and stimulation parameters. Amygdalohippocampal DBS has yielded a significant decrease of seizure counts and interictal EEG abnormalities during long-term follow-up. Data from pilot studies suggest that chronic DBS for epilepsy may be a feasible, effective, and safe procedure. Further trials with larger patient populations and with controlled, randomized, and closed-loop designs should now be initiated. Further progress in understanding the mechanism of action of DBS for epilepsy is a necessary step to making this therapy more efficacious and established. Key Words: Refractory epilepsy, electrical stimulation, neurostimulation, vagus nerve stimulation, hippocampal stimulation, epilepsy surgery.

\section{INTRODUCTION}

Neurostimulation is a treatment modality in which electrical pulses are administered directly to or in the neighborhood of nerve tissue in order to manipulate a pathological substrate and to achieve a symptomatic or even curative therapeutic effect. The several types of neurostimulation differ in the part of the nervous system affected and in the way the stimulation is administered.

Electrical stimulation of the tenth cranial nerve, or vagus nerve stimulation (VNS), is an extracranial form of stimulation that was developed in the 1980s and is currently routinely and worldwide available. Through an implanted device and electrode, electrical pulses are administered to the afferent fibers of the left vagus nerve in the neck. Such VNS is indicated in patients with refractory epilepsy who are unsuitable candidates for epilepsy

Address correspondence and reprint requests to: Prof. Dr. Paul Boon, Reference Center for Refractory Epilepsy, Laboratory for Clinical and Experimental Neurophysiology, Department of Neurology, 1K12IA, Ghent University Hospital, De Pintelaan 185, B-9000 Ghent, Belgium. E-mail: Paul.Boon@UGent.be. surgery or who have had insufficient benefit from such a treatment. ${ }^{1}$ Transcranial magnetic stimulation is another form of extracranial neurostimulation. A coil that transmits magnetic fields held over the scalp allows a noninvasive evaluation of separate excitatory and inhibitory functions of the cerebral cortex. Repetitive transcranial magnetic stimulation is thought to modulate the excitability of cortical networks, ${ }^{2}$ and it is currently under investigation as a treatment option for refractory epilepsy.

In intracerebral neurostimulation, stimulation electrodes are targeted into intracerebral structures for deep brain stimulation (DBS) or placed over the cortical convexity for cortical stimulation. DBS for neurological and psychiatric diseases is not new. DBS has been extensively used in movement disorders and for pain. ${ }^{3,4}$ Several other indications, such as obsessive compulsive behavior and cluster headache, are currently being investigated with promising results. ${ }^{5,6}$ In the past, combined DBS and cortical stimulation of different brain structures such as the cerebellum, the locus coeruleus, and the thalamus have been performed. This was done 
mostly in patients with spasticity or psychiatric disorders who had comorbid epilepsy, but the technique was not fully explored or developed into an efficacious treatment option for seizures. ${ }^{7-10}$ The vast progress in biotechnology, along with experience in other neurological diseases over the past 10 years, has led to a renewed interest in intracerebral stimulation for epilepsy. A few epilepsy centers around the world have recently reinitiated trials with DBS in the thalamus, the subthalamic nucleus, and the caudate nucleus. ${ }^{11-15}$

The present review focuses on VNS and hippocampal DBS as a treatment for epilepsy. The precise mechanism of action by which VNS exerts its antiepileptic effect is unknown. Optimal candidates for VNS have not been identified. About one third of patients undergoing VNS will eventually not benefit from the intervention. Increased insight into the mechanism of action may help to identify responders and so increase clinical efficacy. Inversely, the identification of predictive factors for positive clinical outcome may further elucidate the mechanism of action of VNS.

Compared with VNS, the more invasive DBS treatment option has been investigated only more recently. As with VNS, the precise mechanism of action and the ideal candidates for this treatment option are currently unidentified. Moreover, it is unknown which intracerebral structures should be targeted to achieve optimal clinical efficacy.

Two major strategies can be distinguished. One approach is to target crucial central nervous system structures that are considered to have a pacemaker, triggering, or gating role in the epileptogenic network-such as the thalamus or the subthalamic nucleus. ${ }^{16}$ Another approach is to interfere with the ictal onset zone proper. This implies identification of the ictal onset zone, a process that sometimes requires implantation with intracranial electrodes. ${ }^{17}$ At Ghent University Hospital, this latter approach was chosen.

Electrical pulses applied in VNS and DBS are defined by stimulation parameters (e.g., output current, frequency, pulse width, duty cycle). The parameters chosen are based first on what is known to be safe and second on what is believed to be potentially efficacious. For VNS, the so-called optimal or standard stimulation parameters have been identified on the basis of animal experiments. $^{18}$

For DBS, little information on efficacious stimulation parameters is available from limited human or animal epilepsy experiments. A range of potentially efficacious and safe stimulation parameters have been identified from previous DBS studies in other intracerebral targets $^{7-15}$ and from studies in patients with movement disorders. $^{19}$

\section{VAGUS NERVE STIMULATION}

\section{Clinical efficacy and safety}

Randomized controlled trials. The first descriptions of the implantable VNS Therapy (Cyberonics, Houston, TX) system for electrical stimulation of the vagus nerve in humans appeared in the literature in the early 1990s. ${ }^{20,21}$

At the same time, initial results were reported from two single-blinded pilot clinical trials (phase I trials EO1 and EO2) in a small group of patients with refractory complex partial seizures who had been implanted since November 1988 in three epilepsy centers in the United States. $^{21-25}$ In $9 / 14$ patients, treated for 3 to 22 months, a reduction in seizure frequency of at least $50 \%$ was observed. ${ }^{21}$ One of the patients was seizure-free for more than 7 months. Complex partial seizures, simple partial seizures, and secondary generalized seizures were affected. ${ }^{23}$ A reduction in frequency, duration and intensity of seizures lagged 4 to 8 weeks after the initiation of treatment. ${ }^{22}$

In 1993, Uthman et al. ${ }^{26}$ reported the long-term results from the EO1 and EO2 study. In 14 patients who had been treated for 14 to 35 months, there was a mean reduction in seizure frequency of $46 \%$. Five patients had a seizure reduction of at least $50 \%$, of whom 2 experienced long-term seizure freedom. In none of the patients did VNS induce seizure exacerbation. In the meantime, two prospective multicenter $(n=17)$ double-blind randomized studies (EO3 and EO5) were started, with patients from centers in the United States $(n=12)$ and Canada $(n=1)$, as well as in Europe $(n=4) .^{27-31}$

In these two studies, patients older than 12 years of age with partial seizures were randomized to a HigH or Low stimulation paradigm. The parameters in the $\mathrm{HIGH}$ stimulation group (output: gradual increase up to 3.5 $\mathrm{mA}, 30 \mathrm{~Hz}, 500 \mu \mathrm{s}, 30 \mathrm{~s}$ on, $5 \mathrm{~min}$ off) were those believed to be efficacious based on animal data and the initial human pilot studies. Because patients can sense stimulation, the Low stimulation parameters (output: single increase to point of patient perception, no further increase, $1 \mathrm{~Hz}, 130 \mu \mathrm{s}, 30 \mathrm{~s}$ on, 3 hours off) were chosen to provide some sensation to the patient, to protect the blinding of the study. Low stimulation parameters were believed to be less efficacious, and the patients in this group represented an active control group.

The results of EO3 in 113 patients were promising, with a $24 \%$ decrease in seizures in the HiGH stimulation group versus $6 \%$ decrease in the Low stimulation group after 3 months of treatment. ${ }^{28-30}$ The number of patients was insufficient to achieve Food and Drug Administration (FDA) approval, however, which led to the EO5 study in the United States. This study included 198 patients: 94 patients in the HigH stimulation group had a 
$28 \%$ decrease in seizure frequency versus $15 \%$ in patients in the Low stimulation group. ${ }^{31}$

Clinical trials with long-term follow-up. The controlled EO3 and EO5 studies had their primary efficacy endpoint after 12 weeks of VNS. Patients who ended the controlled trials were offered enrollment in a long-term (1-3 years of follow-up) prospective efficacy and safety study. Patients belonging to the Low stimulation groups were crossed over to HIGH stimulation parameters. In all published reports on these long-term results, increased efficacy with longer treatment was found. ${ }^{32-36}$ In these open extension trials, the mean reduction in seizure frequency increased up to $35 \%$ at 1 year and up to $44 \%$ at 2 years of follow-up. After that, improved seizure control reached a plateau. ${ }^{35}$

In the following years, other large prospective clinical trials were conducted in different epilepsy centers worldwide. In Sweden, long-term follow-up in the largest patient series $(n=67)$ in one center not belonging to the sponsored clinical trials at that time, reported similar efficacy rates, with a mean decrease in seizure frequency of $44 \%$ in patients treated up to 5 years. ${ }^{37} \mathrm{~A}$ joint study of two epilepsy centers in Belgium and in the United States included 118 patients, with a minimum follow-up duration of 6 months; they found a mean reduction in monthly seizure frequency of $55 \% .{ }^{38}$ In China, a mean seizure reduction of $40 \%$ was found in 13 patients after 18 months of VNS. ${ }^{39}$

An open-label retrospective study evaluated long-term outcome in seven different epilepsy centers in Belgium: 138 patients with a follow-up of at least 12 months had an overall reduction in mean monthly seizure frequency of $51 \%$ and a responder rate of $59 \% .{ }^{40}$

At Ghent University Hospital, successful treatment of status epilepticus (SE) with VNS was reported. ${ }^{41}$ In this case, a 7-year-old girl presented with a refractory nonconvulsive SE. A vagus nerve stimulator was placed after 11 days of thiopental-induced coma. Electroencephalography showed normalization 1 week after the start of VNS, and the patient experienced a sustained seizure-free outcome after a follow-up of more than 13 months.

A few other case reports describing the use of VNS for refractory SE in pediatric and adult patients are available. Malik et al. ${ }^{42}$ described the case of three children with pharmacoresistant SE who were successfully treated with VNS; it was not specified whether the status was convulsive or nonconvulsive in these patients. Winston et al. ${ }^{43}$ described the case of a 13 -year-old boy in whom VNS interrupted a convulsive SE immediately after stimulation was started. Patwardan et al. ${ }^{44}$ described the case of a 30-year-old man with medically intractable seizures due to severe allergic reactions to multiple antiepileptic drugs (AEDs), with subsequent evolution into refractory SE. He underwent VNS treatment after nearly 9 days of barbiturate-induced coma. Stimulation was initiated in the operating room; in the following days, EEG revealed resolution of previously observed periodic lateralized epileptiform discharges with the stimulator programmed at $1 \mathrm{~mA}$ and a duty cycle of $30 \mathrm{~s}$ on and $3 \mathrm{~min}$ off. The patient became seizure-free. ${ }^{44}$ Zimmerman et al. ${ }^{45}$ described the cases of three adult patients in whom refractory nonconvulsive SE due to AED withdrawal was treated with VNS. After implantation of the device, stimulation output was rapidly increased to $3 \mathrm{~mA}$; time to termination of the SE was 3 to 5 days.

\section{Safety, side effects, and tolerability}

Safety concerns with regard to VNS treatment can be approached from different perspectives. Because the device needs to be implanted, a surgical intervention is required. The effects of delivering current to nerve tissue need to be considered, because this might provoke changes in innervated organs and result in acute or chronic side effects. Patients with refractory epilepsy are often young people. The implanted device and wires have to be examined for MRI compatibility.

The classical surgical technique for VNS has been described in detail by several authors. ${ }^{46-48}$ Adverse cosmetic effects have already been reduced since the production of the smaller Model 101, and further improvement is expected once the Model 103 Generator Demipulse and Model 104 Generator Demipulse Duo become widely available.

Ramping up and long-term stimulation. For therapeutic purposes, VNS aims at stimulating vagal afferent fibers. There are widespread connections from the vagus nerve to the central nervous system. Through these connections, efficacious stimulation parameters may also induce other central nervous system side effects. Moreover, selectively stimulating afferent fibers is difficult, and approximately $20 \%$ of the fibers in the cervical part of the vagus nerve are efferent. These fibers innervate thoracoabdominal organs - thus the potential for serious side effects when these fibers are stimulated. ${ }^{29}$ Certain side effects related to undesired stimulation of nerve fibers might be immediately perceptible by the patient. The main efferent innervation of the vagus nerve serves to monitor and modulate visceral activity. These autonomic processes are usually not perceived by the patient. There may also be side effects specifically related to chronic stimulation that will cause symptoms and become clinically apparent only after long-term treatment.

The most prominent and consistent sensation in patients when the vagus nerve is stimulated for the first time, even at low output current levels, is a tingling sensation in the throat and hoarseness of the voice. The tingling sensation may be due to secondary stimulation of the superior laryngeal nerve, which branches off from the vagus nerve superior to the location of the implanted 
electrode but travels along the vagus nerve in the carotid sheath. ${ }^{49}$ The superior laryngeal nerve carries sensory fibers to the laryngeal mucosa. Stimulation of the recurrent laryngeal nerve that branches off distally from the location of the electrode and carries motor ( $\mathrm{A} \alpha)$ fibers to the laryngeal muscles causes the stimulation-related hoarseness. ${ }^{50,51}$ Fiber-optic laryngoscopy and videostroboscopic examination have shown left vocal cord adduction (tetanic contraction) during stimulation at $30 \mathrm{~Hz}$ or higher. ${ }^{51-55}$ These stimulation-related side effects are dose dependent, which means that higher amplitudes, higher frequencies, and wider pulse widths are associated with more intense sensations and voice changes. ${ }^{26}$

With regard to side effects related to stimulation of vagal efferents, effect on heart rate has been a major concern. The stimulation electrode is always implanted on the left vagus nerve, which is believed to contain fewer sinoatrial fibers than the right.

In the long-term extension trials, the most frequent side effects were hoarseness (in 19\% of patients) and coughing (in $5 \%$ of patients) at 2 years follow-up and shortness of breath (in $3 \%$ of patients) at 3 years. ${ }^{35}$ There was a clear trend toward diminishing side effects over the 3-year stimulation period. Ninety-eight percent of the symptoms were rated mild or moderate by the patients and the investigators. ${ }^{56}$ Side effects can usually be resolved by decreasing stimulation parameters. Central nervous system side effects typically seen with AEDs were not reported. After 3 years of treatment, $72 \%$ of patients were still on the treatment. ${ }^{35}$ The most frequent reason for discontinuation was lack of efficacy. Holter monitoring in a sample of patients of the EO4 study showed no clinically symptomatic changes. Pulmonary function testing was performed in 124 patients, with no change between baseline and long-term treatment. ${ }^{34}$ Even though initial studies showed no clinical effect on heart rate, occurrence of bradycardia and ventricular asystole during intraoperative testing of the device (stimulation parameters: $1 \mathrm{~mA}, 20 \mathrm{~Hz}, 500 \mu \mathrm{s}, \sim 17 \mathrm{~s}$ ) have been reported in a few patients. None of the reported patients had a history of cardiac dysfunction, nor did they show abnormal cardiac testing after surgery.

Tatum et al. ${ }^{57}$ described the cases of four patients who experienced ventricular asystole intraoperatively during device testing. In three patients, the implantation procedure was aborted. In one patient, a rechallenge of stimulation with incremental increases from $0.25 \mathrm{~mA}$ to 1 $\mathrm{mA}$ did not reveal a reappearance of bradycardia. Implantation was completed and no further cardiac events were noticed after start of VNS. Asconapé et al. ${ }^{58} \mathrm{de}-$ scribed the case of a single patient who developed asystole during intraoperative device testing. After removal of the device, the patient recovered completely. Ali et al. ${ }^{59}$ described three similar cases. Cardiac rhythm strips were available and showed a regular p-wave (i.e., atrial rhythm) with no ventricular activity indicating a complete AV nodal block. In two of these patients the device was subsequently removed. In one patient the device was left in place without experiencing any other adverse events after start of VNS. ${ }^{59}$ Andriola et al. ${ }^{60}$ described the cases of three patients who experienced an asystole during intraoperative lead testing and who were subsequently chronically stimulated. Ardesch et al. ${ }^{61}$ described the cases of three patients with intraoperative bradycardia and subsequent uneventful stimulation.

Possible hypotheses with regard to the underlying cause are inadvertent placement of the electrode on one of the cervical branches of the vagus nerve or indirect stimulation of these branches, reversal of the polarities of the electrode (which would lead to primary stimulation of efferent instead of afferent fibers), indirect stimulation of cardiac branches, activation of afferent pathways affecting higher autonomic systems or of the parasympathetic pathway with an exaggerated effect on the atrioventricular node, technical malfunctioning of the device or idiosyncratic reactions. The contributing role of specific AEDs should be further investigated. Suggested steps to be taken in the operating room in case of bradycardia or asystole during generator and lead impedance testing have been formulated by Asconapé et al. ${ }^{58}$ Adverse cardiac complications at start or during ramping-up of the stimulation intensity have not been observed. ${ }^{29}$ Very recently, one case report described a late onset bradyarrhythmia after 2 years of vagus nerve stimulation. $^{62}$

\section{MRI}

Most patients with refractory epilepsy who are treated with VNS have previously undergone MRI during the presurgical evaluation. It is not uncommon for such patients to require MRI after VNS implantation, for further monitoring of underlying neurological diseases (e.g., in case of unexplained seizure frequency increase, follow-up of intracranial lesions, or for MRI indications as in the general population). Based on laboratory testing using a phantom to simulate a human body, the VNS Therapy system device is labeled MRI compatible when used with a send-and-receive head coil. ${ }^{63}$ Another report of MRI-compatible safety indicated also that there was no significant image distortion. ${ }^{64}$

A retrospective analysis of 27 MRI scans performed in 25 patients in 12 different centers confirmed the findings from the experimental set-up in a clinical series. All patients were scanned on a 1.5-T machine. On one occasion a body coil was used. Three scans were performed with the stimulator in the ON mode. One adult patient reported a mild voice change for several minutes, and one reported chest pain and claustrophobia; the remaining 23 patients reported no discomfort around the lead or 
the generator. It was concluded that MRI is safe as long as guidelines stated in the physician's manual of the implanted device are followed.

In these guidelines, it is suggested to program the pulse generator output current and the magnet output current to $0 \mathrm{~mA}$. On one occasion, this led to the occurrence of a generalized status epilepticus in a patient who was well controlled with an output current of $2 \mathrm{~mA}^{65}$ The authors of the report recommend that intravenous access should be obtained and a benzodiazepine should be either available or preadministered in patients with a well-defined response who undergo elective MRI and in whom the generator is acutely programmed to $0 \mathrm{~mA}$.

Functional MRI (fMRI) is a recently developed technique that allows noninvasive evaluation of cerebral functions, such as finger movements and language. ${ }^{66}$ It has been widely used for research, but is currently increasingly applied to evaluate functional tissue in the neighborhood of lesions before resective surgery and also for assessing language dominance in the presurgical evaluation of epilepsy patients. ${ }^{66,67}$ When fMRI in patients with VNS is used for research purposes to evaluate VNS-induced changes in cerebral blood flow, scanning should be performed in the ON mode. To prevent the device from being turned off during scanning, an adjustment in the surgical positioning of the device is necessary. The device should be positioned so that the electrode pins that are plugged into the generator are parallel instead of perpendicular to the long axis of the body. ${ }^{68}$ There have been several studies in patients treated with VNS for epilepsy as well as for depression showing that fMRI is safe and feasible. ${ }^{69-73}$ These studies were performed to elucidate the mechanism of action of VNS (for discussion, see next section).

The use of body coils may be indicated in patients requiring spinal MRI. Placing a cold pack (water and ammonium nitrate) over the left side of the patient's neck protected the vagus nerve in three children from heat, which can theoretically be generated when using the body coil. ${ }^{74}$

When removal of the electrode is indicated (e.g., due to insufficient efficacy), complete removal is recommended over cutting the distal edges and leaving the electrode in place. ${ }^{75}$ Full removal allows potential future MRI with body coils. Heating of the electrode is related to the lead length. If full removal of the electrode is difficult, then the leads should be cut to less than $10 \mathrm{~cm}$.

In several of our patients, uneventful MRI was performed according to the prescribed precautions. In one patient with frequent simple partial seizures successful and uneventful fMRI was performed with the stimulator in the OfF mode.

\section{Mechanism of action}

Since the first human implant of the VNS Therapy device in 1989, more than 50,000 patients have been treated with VNS worldwide. As for many antiepileptic treatments, clinical application of VNS preceded the elucidation of its mechanism of action. After a limited number of animal experiments in dogs and monkeys investigating safety and efficacy, the first human trial was performed. $^{22}$ The basic hypothesis on the mechanism of action was based on the knowledge that the tenth cranial nerve afferent fibers have numerous projections within the central nervous system and that in this way action potentials generated in vagal afferent fibers have the potential to affect the entire organism. ${ }^{76}$ To date, the precise mechanism of action of VNS and how it suppresses seizures remain to be elucidated.

Research has been performed directed toward investigating the antiseizure, antiepileptic, and potentially antiepileptogenic properties of VNS, as well as toward the identification of the fibers, intracranial structures, and neurotransmitter systems involved. Animal experiments and research in humans treated with VNS have comprised electrophysiological studies (EEG, EMG, and evoked potentials), functional anatomic brain imaging studies (positron emission tomography, single-photon emission computed tomography, fMRI, c-fos, and densitometry), and neuropsychological and behavioral studies. Interesting clues concerning the mechanism of action of VNS have arisen from the extensive clinical experience with VNS. More recently, the role of the vagus nerve in the immune system has been investigated.

Based on the extensive body of research on the mechanism of action, it is conceivable that effective stimulation in humans is primarily mediated by afferent vagal A and B fibers. ${ }^{77,78}$ Unilateral stimulation influences both cerebral hemispheres, as shown in several functional imaging studies. ${ }^{79,80}$ Crucial brainstem and intracranial structures have been identified; these include the locus coeruleus, the nucleus of the solitary tract, the thalamus, and limbic structures. ${ }^{81-84}$

Neurotransmitters playing a role may involve the major inhibitory neurotransmitter GABA, but also serotoninergic and adrenergic systems. ${ }^{85,86}$ Recently, Neese et al. ${ }^{87}$ found that VNS following experimental brain injury in rats protects cortical GABAergic cells from death. A single-photon emission computed tomography study in humans before and after 1 year of VNS showed a normalization of $\mathrm{GABA}_{\mathrm{A}}$ receptor density in the individuals with a clear therapeutic response to $\mathrm{VNS} .{ }^{88}$ Follesa et al. ${ }^{89}$ found an increase of norepinephrine concentration in the prefrontal cortex of the rat brain after acute VNS. An increased norepinephrine concentration after VNS has also been measured in the hippocampus ${ }^{90}$ and amygdala. ${ }^{91}$ 
Currently, VNS as a neuroimmunomodulatory treatment is being explored. Because the vagus nerve plays a critical role in the signalization and modulation of inflammatory processes, the so-called anti-inflammatory pathway, this could represent a new modality in the mechanism of action of VNS for epilepsy. ${ }^{92,93}$

Early animal experiments in acute seizure models suggest an antiseizure effect of VNS. In our own group, VNS significantly increased the seizure threshold for focal motor seizures in the cortical stimulation model. ${ }^{94}$ Evidence also exists, from the human literature, that VNS may exert an acute abortive effect: the magnet feature allows a patient to terminate an upcoming seizure. ${ }^{95}$ Also, a few case reports describe the use of VNS for refractory SE in pediatric and adult patients. ${ }^{41,42}$ A recent study investigated the effects of acute VNS on cortical excitability by using transcranial magnetic stimulation. ${ }^{96}$

The fact that seizures recur after the end of battery life has been reached is a strong argument against an antiepileptogenic effect of VNS. Nonetheless, as progress has been made in the development of more relevant animal models for epilepsy, the antiepileptogenic potential of neurostimulation in general is being fully explored and some promising results have been reported-in, for example, in the kindling model. ${ }^{97,98}$ The basis for the combined acute and more chronic effects of VNS most likely involves recruitment of different neuronal pathways and networks. The more chronic effects are thought to be a reflection of modulatory changes in subcortical site-specific synapses with the potential to influence larger cortical areas. In the complex human brain, these neuromodulatory processes require time to build up. Once installed, certain antiepileptic neural networks may be more easily recruited - for example by changing stimulation parameters, which may then be titrated to the individual need of the patient. This raises the hope for eliciting the potential antiepileptogenic properties of VNS using long-term optimized stimulation parameters.

\section{HIPPOCAMPAL DBS}

\section{Clinical efficacy and safety}

Electrical seizure onset in the amygdala and hippocampus is the key feature of the medial temporal lobe epilepsy syndrome. ${ }^{99}$ Acute DBS in medial temporal lobe structures for control of seizures has been described. ${ }^{100}$ In a small number of patients with complex partial seizures requiring invasive video-EEG monitoring for localizing purposes, unilateral DBS decreased interictal and ictal epileptic activity during a 2-week period using temporary depth electrodes. The recording electrodes that were used for invasive video-EEG monitoring are unsuitable for long-term DBS and had to be removed. Subsequently, all patients underwent a temporal lobectomy.
Animal studies showed abortive effects on epileptic activity when electrical fields were applied to hippocampal slices. ${ }^{101}$ In vivo studies in rats showed that electrical stimuli applied after a kindling stimulus (called quenching) can delay the development of the kindling process. ${ }^{102}$ Bragin et al. ${ }^{103}$ and Velisek et al. ${ }^{104}$ found that repeated stimulation of the hippocampal perforant path in the kainate rat model significantly reduced seizures.

Performing chronic DBS implies removal of recording electrodes and replacement by chronic DBS electrodes. Because the purpose is to stimulate the ictal onset zone, replacement of electrodes should be anatomically as accurate as possible. Even with currently available neuronavigation technology, positioning of a second electrode in exactly the same position as the initial one is difficult. We have therefore studied the feasibility of recording intracranial EEG activity for localizing purposes and subsequent long-term DBS of the identified ictal onset zone using the same electrodes, to evaluate the long-term efficacy and safety of chronic DBS in medial temporal lobe structures, and also to investigate the feasibility of using chronic DBS electrodes for the localization of the ictal onset zone prior to DBS to avoid an additional invasive procedure.

An initial pilot study was performed to demonstrated proof-of-concept. ${ }^{105}$ The number of patients in the pilot study was increased, and long-term follow-up was reported. ${ }^{106}$ The study prospectively evaluated the efficacy of long-term ${ }^{100}$ in medial temporal lobe structures in patients with medial temporal lobe epilepsy; 12 consecutive patients with refractory medial temporal lobe epilepsy were included. The protocol included invasive video-EEG monitoring for ictal onset localization and evaluation for subsequent stimulation of the ictal onset zone. Side effects and changes in seizure frequency were carefully monitored. Of the 12 patients, 10 patients underwent chronic medial temporal lobe DBS and 2 patients underwent temporal lobectomy. After mean follow-up of 31 months (range, 12-52 months), 1 of the 10 stimulated patients was seizure free ( $>1$ year), 1 had a $>90 \%$ reduction in seizure frequency, 5 had a $>50 \%$ reduction in seizure frequency, and 2 had a seizure frequency reduction of $30 \%$ to $49 \%$; the 1 remaining patient was a nonresponder.

None of the patients reported side effects. In one patient, MRI showed asymptomatic intracranial hemorrhages along the trajectory of the DBS electrodes. None of the patients showed changes in clinical neurological testing. The two patients who underwent temporal lobectomy were seizure free $(>1$ year), with no change in AEDs and no side effects reported.

This open pilot study demonstrates the potential efficacy of long-term DBS in medial temporal lobe structures, which should now be further confirmed by multicenter randomized controlled trials. CoRaStiR is an 
ongoing multicenter study in Belgium and Germany including patients with unilateral hippocampal sclerosis who will be randomized to unilateral hippocampal DBS or amygdalohippocampal resection. Early experience with responsive stimulation (closed-loop stimulation) has shown promising results. ${ }^{107}$ The results of a large multicenter U.S. trial are currently pending, as are results from another large randomized controlled U.S. trial, investigating the safety and efficacy of anterior nucleus stimulation.

\section{Mechanism of action}

The mechanism of action of DBS in reducing seizures remains unclear. Some authors support the hypothesis that actual stimulation is not necessary to achieve efficacy and claim that efficacy is based on the lesion provoked by the insertion of the electrode (the so-called microthalamotomy effect). ${ }^{15}$ Furthermore, prolonged seizure control in patients who underwent invasive recording with conventional electrodes has been described. ${ }^{108}$ Blinded randomization of patients to ON and OfF stimulation paradigms after implantation during follow-up ( $>6$ months) may clarify this issue, and could also simultaneously clarify the potential effect of sham stimulation due to an implanted device.

It is also possible that DBS acts through local inhibition induced by current applied to nuclei that are involved in propagating, sustaining, or triggering epileptic activity in a specific CNS structure (i.e., reversible functional lesion). Apart from this local inhibition, the mechanism of action of DBS may be based on the effect on projections leaving from the area of stimulation to other central nervous structures. This may be the most likely hypothesis when crucial structures in epileptogenic networks are involved. Nonetheless, considering that the medial temporal lobe structures are also potentially involved in these networks, it may be that targeting the ictal focus may also affect the epileptogenic network.

\section{CONCLUSION}

Patients with refractory epilepsy present a particular therapeutic challenge. Vagus nerve stimulation for epilepsy has been demonstrated to be moderately efficacious, comparable to new AEDs, and safe. The efficacy of VNS in less severely affected populations remains to be evaluated. The current consensus on efficacy is that one third of patients have a considerable improvement in seizure control with a reduction in seizure frequency of at least $50 \%$, one third of patients experience a worthwhile reduction of seizure frequency between $30 \%$ and $50 \%$, and the remaining one third of patients experience little or no effect. Vagus nerve stimulation seems equally efficient for children. Unlike treatment with AEDs, effi- cacy of VNS has a tendency to improve with longer duration of treatment, up to 18 months postoperatively.

Further analysis of larger patient groups and better insight in the mode of action may help to identify the best responders and guide the search for optimal stimulation parameters, resulting in further improvement of clinical efficacy. DBS for epilepsy is beyond the stage of proof-of-concept, but still needs thorough evaluation in confirmatory pilot studies and pivotal randomized controlled trials before it can be offered to a larger patient population. The most adequate targets and stimulation parameters still need to be identified. For patients who are less suitable candidates for epilepsy surgery, DBS may become a valuable alternative.

\section{Disclosure}

P.B. is a senior clinical researcher with the Fund for Scientific Research Flanders and is supported by grants from the European Union (6FP), the Fund for Scientific Research Flanders (FWO), the Ghent University Research Fund (BOF), and the Clinical Epilepsy Grant of Ghent University Hospital. K.V. is a Ghent University Research Fund Professor (BOF-ZAP). Medtronic supported part of this research by providing free inplantable devices and electrodes for the hippocampal DBS pilot study. Both authors have received fees for presentations and consultancy from Cyberonics and Medtronic.

\section{REFERENCES}

1. Ben-Menachem E. Vagus-nerve stimulation for the treatment of epilepsy. Lancet Neurol 2002;1:477-482.

2. Tassinari CA, Cincotta M, Zaccara G, Michelucci R. Transcranial magnetic stimulation and epilepsy. Clin Neurophysiol 2003;114: 777-798.

3. Pollak P, Fraix V, Krack P, et al. Treatment results: Parkinson's disease. Mov Disord 2002;17 Suppl 3:S75-S83.

4. Ngyuen JP, Lefauche[u]r JP, Le Guerinel C, et al. Motor cortex stimulation in the treatment of central and neuropathic pain. Arch Med Res 2000;31:263-265.

5. Nuttin B, Cosyns P, Demeulemeester H, Gybels J, Meyerson B. Electrical stimulation in anterior limbs of internal capsules in patients with obsessive-compulsive disorder. Lancet 1999 30; 354:1526.

6. Leone M, Franzini A, Broggi G, Bussone G. Hypothalamic deep brain stimulation for intractable chronic cluster headache: a 3-year follow-up. Neurol Sci 2003;24 Suppl 2:S143-S145.

7. Cooper IS. Cerebellar stimulation in man. New York: Raven Press, 1978.

8. Wright GD, McLellan DL, Brice JG. A double-blind trial of chronic cerebellar stimulation in twelve patients with severe epilepsy. J Neurol Neurosurg Psychiatry 1984;47:769-774.

9. Upton AR, Cooper IS, Springman M, Amin I. Suppression of seizures and psychosis of limbic system origin by chronic stimulation of anterior nucleus of the thalamus. Int J Neurol 19851986;19-20:223-230.

10. Feinstein B, Gleason CA, Libet B. Stimulation of locus coeruleus in man: Preliminary trials for spasticity and epilepsy. Stereotact Funct Neurosurg 1989;52:26-41.

11. Fisher RS, Uematsu S, Krauss GL, et al. Placebo-controlled pilot study of centromedian thalamic stimulation in treatment of intractable seizures. Epilepsia 1992;33:841-851.

12. Velasco F, Velasco M, Velasco AL, Jimenez F, Marquez I, Rise M. Electrical stimulation of the centromedian thalamic nucleus in control of seizures: long-term studies. Epilepsia 1995;36:63-71. 
13. Chkhenkeli SA, Chkhenkeli IS. Effects of therapeutic stimulation of nucleus caudatus on epileptic electrical activity of brain in patients with intractable epilepsy. Stereotact Funct Neurosurg 1997;69:221-224.

14. Chabardes S, Kahane P, Minotti L, Koudsie A, Hirsch E, Benabid AL. Deep brain stimulation in epilepsy with particular reference to the subthalamic nucleus. Epileptic Disord 2002;4 Suppl 3:S83S93.

15. Hodaie M, Wennberg RA, Dostrovsky JO, Lozano AM. Chronic anterior thalamus stimulation for intractable epilepsy. Epilepsia 2002;43:603-608

16. Iadarola MJ, Gale K. Substantia nigra: site of anticonvulsant activity mediated by gamma-aminobutyric acid. Science 1982; 218:1237-1240.

17. King D, Spencer S. Invasive electroencephalography in mesial temporal lobe epilepsy. J Clin Neurophysiol 1995;12:32-45.

18. Woodbury JW, Kinghorn EW. Vagus nerve stimulation for control of epilepsy in humans: suggested new parameters. Epilepsia 1998;39 Suppl 6:194 (abstract).

19. Benabid AL, Koudsie A, Benazzouz A, et al. Deep brain stimulation of the corpus luysi (subthalamic nucleus) and other targets in Parkinson's disease: extension to new indications such as dystonia and epilepsy. J Neurol 2001;248(S3):37-47.

20. Terry R, Tarver WB, Zabara J. An implantable neurocybernetic prosthesis system. Epilepsia 1990;31 Suppl 2:S33-S37.

21. Terry RS, Tarver WB, Zabara J. The implantable neurocybernetic prosthesis system. Pacing Clin Electrophysiol 1991;14:86-93.

22. Uthman BM, Wilder BJ, Hammond EJ, Reid SA. Efficacy and safety of vagus nerve stimulation in patients with complex partial seizures. Epilepsia 1990;31 Suppl 2:S44-S50.

23. Penry JK, Dean JC. Prevention of intractable partial seizures by intermittent vagal stimulation in humans: preliminary results. Epilepsia 1990;31 Suppl 2:S40-S43.

24. Hammond EJ, Uthman BM, Reid SA, Wilder BJ, Ramsay RE. Vagus nerve stimulation in humans: neurophysiological studies and electrophysiological monitoring. Epilepsia 1990;31 Suppl 2:S51-S59.

25. Wilder BJ, Uthman BM, Hammond EJ. Vagal stimulation for control of complex partial seizures in medically refractory epileptic patients. Pacing Clin Electrophysiol 1991;14:108-115.

26. Uthman B, Wilder BJ, Penry JK, et al. Treatment of epilepsy by stimulation of the vagus nerve. Neurology 1993;43:1338-1345.

27. Holder LK, Wernicke JF, Tarver WB. Treatment of refractory partial seizures: preliminary results of a controlled study. Pacing Clin Electrophysiol 1992;15:1557-1571.

28. Ben-Menachem E, Mañon-Espaillat R, Ristanovic R, et al.; First International Vagus Nerve Stimulation Study Group. Vagus nerve stimulation for treatment of partial seizures: 1. A controlled study of effect on seizures. Epilepsia 1994;35:616-626.

29. Ramsay RE, Uthman BM, Augustinsson LE, et al.; First International Vagus Nerve Stimulation Study Group. Vagus nerve stimulation for treatment of partial seizures: 2. Safety, side-effects, and tolerability. Epilepsia 1994;35:627-636.

30. The Vagus Nerve Stimulation Study Group. A randomized controlled trial of chronic vagus nerve stimulation for treatment of medically intractable seizures. Neurology 1995;45:224-230.

31. Handforth A, DeGiorgio CM, Schachter SC, et al. Vagus nerve stimulation therapy for partial onset seizures: a randomized, active control trial. Neurology 1998;51:48-55.

32. Holder L, Wernicke JF, Tarver WB. Long-term follow-up of 37 patients with refractory partial seizures treated with vagus nerve stimulation. J Epilepsy 1993;6:206-214.

33. George R, Salinsky M, Kuzniecky R, et al.; First International Vagus Nerve Stimulation Study Group. Vagus nerve stimulation for treatment of partial seizures: 3. Long-term follow-up on first 67 patients exiting a controlled study. Epilepsia 1994;35:637643.

34. Salinsky MC, Uthman BM, Ristanovic RK, Wernicke JF, Tarver WB; Vagus Nerve Stimulation Study Group. Vagus nerve stimulation for the treatment of medically intractable seizures: results of a 1-year open-extension trial. Arch Neurol 1996;53:11761180 .
35. Morris GL, Mueller WM. Long-term treatment with vagus nerve stimulation in patients with refractory epilepsy [Erratum in: Neurology 2000;54:1712]. Neurology 1999;53:1731-1735.

36. DeGiorgio CM, Schachter SC, Handforth A, et al. Prospective long-term study of vagus nerve stimulation for the treatment of refractory seizures. Epilepsia 2000;41:1195-1200.

37. Ben-Menachem E, Hellström K, Waldton C, Augustinsson LE. Evaluation of refractory epilepsy treated with vagus nerve stimulation for up to 5 years. Neurology 1999;52:1265-1267.

38. Vonck K, Thadani V, Gilbert K, et al. Vagus nerve stimulation for refractory epilepsy: a transatlantic experience. J Clin Neurophysiol 2004;21:283-289.

39. Hui AC, Lam JM, Wong KS, Kay R, Poon WS. Vagus nerve stimulation for refractory epilepsy: long-term efficacy and sideeffects. Chin Med J (Engl) 2004;117:58-61.

40. De Herdt V, Boon P, Ceulemans B, et al. Vagus nerve stimulation for refractory epilepsy: a Belgian multicenter study. Eur J Paediatr Neurol 2007;11:261-269.

41. Boon P, De Herdt V, Waterschoot L, et al. Vagus nerve stimulation for refractory status epilepticus. Abstract presented at the 27th International Epilepsy Congress, International League Against Epilepsy (ILAE) and International Bureau for Epilepsy (IBE), Singapore, 2007.

42. Malik S, Hernandez A. Intermittent vagus nerve stimulation in pediatric patients with pharmacoresistant status epilepticus. Epilepsia 2004;4 Suppl 7:155-156 (abstract).

43. Winston KR, Levisohn P, Miller BR, Freeman J. Vagal nerve stimulation for status epilepticus. Pediatr Neurosurg 2001;34: 190-192.

44. Patwardhan RV, Dellabadia J Jr, Rashidi M, Grier L, Nanda A. Control of refractory status epilepticus precipitated by anticonvulsant withdrawal using left vagus nerve stimulation: a case report. Surg Neurol 2005;64:170-173.

45. Zimmerman R, Sirven J, Drazkowski J, Bortz J, Shulman D. High-output current/rapid cycling vagus nerve stimulation for refractory status epilepticus: preliminary results. Epilepsia 2002;43 Suppl 7:286 (abstract).

46. Kemeny AA. Surgical technique in vagus nerve stimulation. In: Schachter SC, Schmidt D, editors. Vagus nerve stimulation. 2nd ed. London, Martin Dunitz 2003:33-48.

47. Reid SA. Surgical technique for implantation of the neurocybernetic prosthesis. Epilepsia 1990;31 Suppl 2:S38-S39.

48. Landy HJ, Ramsay RE, Slater J, Casiano RR, Morgan R. Vagus nerve stimulation for complex partial seizures: surgical technique, safety, and efficacy. J Neurosurg 1993;78:26-31.

49. Claes J, Jaco P. The nervus vagus. Acta Otorhinolaryngol Belg 1986;40:215-241.

50. Banzett RB, Guz A, Paydarfar D, Shea SA, Schachter SC, Lansing RW. Cardiorespiratory variables and sensation during stimulation of the left vagus in patients with epilepsy. Epilepsy Res 1999;35:1-11.

51. Charous SJ, Kempster G, Manders E, Ristanovic R. The effect of vagal nerve stimulation on voice. Laryngoscope 2001;111:2028 2031.

52. Ristanovic RK, Bergen D, Szidon P, Bacon MJ. Documented vagal stimulation-induced ipsilateral vocal cord tetanic contraction. Epilepsia 1992;33 Suppl 3:101 (abstract).

53. Lundy DS, Casiano RR, Landy HJ, Gallo J, Gallo B, Ramsey RE. Effects of vagal nerve stimulation on laryngeal function. J Voice 1993;7:359-364.

54. Zumsteg D, Jenny D, Wieser HG. Vocal cord adduction during vagus nerve stimulation for treatment of epilepsy. Neurology 2000;54:1388-1389.

55. Kersing W, Dejonckere PH, van der Aa HE, Buschman HPJ. Laryngeal and vocal changes during vagus nerve stimulation in epileptic patients. J Voice 2002;16:251-257.

56. Ben-Menachem E. Vagus nerve stimulation, side effects and long-term safety. J Clin Neurophysiol 2001;18:415-418.

57. Tatum WO 4th, Moore DB, Stecker MM, et al. Ventricular asystole during vagus nerve stimulation for epilepsy in humans. Neurology 2000;52:1267-1269.

58. Asconapé JJ, Moore DD, Zipes DP, Hartman LM, Duffell WH Jr. Bradycardia and asystole with the use of vagus nerve stimulation 
for the treatment of epilepsy: a rare complication of intraoperative device testing. Epilepsia 1999;40:1452-1454.

59. Ali II, Pirzada N, Kanjwal Y, et al. Complete heart block with ventricular asystole during left vagus nerve stimulation for epilepsy. Epilepsy Behav 2004;5:768-771.

60. Andriola MR, Rosenweig T, Vlay S, Brook S. Vagus nerve stimulator (VNS): induction of asystole during implantations with subsequent successful stimulation. Epilepsia 2000;41 Suppl 7:223 (abstract).

61. Ardesch JJ, Buschman HP, van der Burgh PH, Wagener-Schimmel LJ, van der Aa HA, Hageman G. Cardiac responses of vagus nerve stimulation: intraoperative bradycardia and subsequent chronic stimulation. Clin Neurol Neurosurg 2007;109:849-852.

62. Amark P, Stödberg T, Wallstedt L. Late onset bradyarrhythmia during vagus nerve stimulation. Epilepsia 2007;48:1023-1024.

63. Nyenhuis JA, Bourland JD, Foster KS, Graber GP, Terry RS, Adkins RA. Testing of MRI compatibility of the cyberonics model $100 \mathrm{NCP}$ and model 300 series lead. Epilepsia 1997;38 Suppl 8:140 (abstract).

64. Benbadis SR, Nyhenhuis J, Tatum WO 4th, Murtagh FR, Gieron M, Vale FL. MRI of the brain is safe in patients implanted with the vagus nerve stimulator. Seizure 2001;10:512-515.

65. Beitinjaneh F, Guido M, Andriola MR. Status epilepticus precipitated by turning off the vagus nerve stimulator for elective brain MRI, a case study. Epilepsia 2002;43 Suppl 7:337-338 (abstract).

66. Achten E, Jackson GD, Cameron JA, Abbott DF, Stella DL, Fabinyi GC. Presurgical evaluation of the motor hand area with functional MR imaging in patients with tumors and dysplastic lesions. Radiology 1999;210:529-538.

67. Deblaere K, Backes WH, Hofman P, et al. Developing a comprehensive presurgical functional MRI protocol for patients with intractable temporal lobe epilepsy: a pilot study. Neuroradiology 2002;44:667-673.

68. Maniker A, Liu WC, Marks D, Moser K, Kalnin A. Positioning of vagal nerve stimulators: technical note. Surg Neurol 2000;53: $178-181$.

69. Sucholeiki R, Alsaadi T, Morris GL, Biswal B. fMRI in patients with vagus nerve stimulation. Epilepsia 1999;40 Suppl 7:181 (abstract).

70. Bohning DE, Lomarev MP, Denslow S, Nahas Z, Shastri A, George MS. Feasibility of vagus nerve stimulation-synchronized blood oxygenation level-dependent functional MRI. Invest Radiol 2001;8:470-479.

71. Sucholeiki R, Alsaadi TM, Morris GL 3rd, Ulmer JL, Biswal B, Mueller WM. fMRI in patients implanted with a vagal nerve stimulator. Seizure 2002;11:157-162.

72. Narayanan JT, Watts R, Haddad N, Labar DR, Li PM, Filippi CG. Cerebral activation during vagus nerve stimulation: a functional MR study. Epilepsia 2002;43:1509-1514.

73. Lomarev M, Denslow S, Nahas Z, Chae JH, George MS, Bohning DE. Vagus nerve stimulation (VNS) synchronized BOLD fMRI suggests that VNS in depressed adults has frequency/dose dependent effects. J Psychiatr Res 2002;36:219-227.

74. Wilfong AA. Body MRI and vagus nerve stimulation. Epilepsia 2002,43 Suppl 7:347 (abstract).

75. Espinosa J, Aiello MT, Naritoku DK. Revision and removal of stimulating electrodes following long-term therapy with the vagus nerve stimulator. Surg Neurol 1999;51:659-664.

76. Berthoud H, Neuhuber WL. Functional and chemical anatomy of the afferent vagal system. Auton Neurosci 2000;85:1-17.

77. Zagon A, Kemeny A. Slow hyperpolarization in cortical neurons: a possible mechanism behind vagus nerve stimulation therapy for refractory epilepsy? Epilepsia 2000;41:1382-1389.

78. Evans MS, Verma-Ahuja S, Naritoku DK, Espinosa JA. Intraoperative human vagus nerve compound action potentials. Acta Neurol Scand 2004;110:232-238.

79. Henry TR, Bakay RAE, Votaw JR, et al. Brain blood flow alterations induced by therapeutic vagus nerve stimulation in partial epilepsy: I. Acute effects at high and low levels of stimulation. Epilepsia 1998;39:983-990.

80. Van Laere K, Vonck K, Boon P, Versijpt J, Dierckx R. Perfusion SPECT changes after acute and chronic vagus nerve stimulation in relation to prestimulus condition and long-term clinical efficacy. J Nucl Med 2002;43:733-744.

81. Naritoku DK, Terry WJ, Helfert RH. Regional induction of fos immunoreactivity in the brain by anticonvulsant stimulation of the vagus nerve. Epilepsy Res 1995;22:53-62.

82. Krahl SE, Clark KB, Smith DC, Browning RA. Locus coeruleus lesions suppress the seizure-attenuating effects of vagus nerve stimulation. Epilepsia 1998;39:709-714.

83. Osharina V, Bagaev V, Wallois F, Larnicol N. Autonomic response and Fos expression in the NTS following intermittent vagal stimulation: importance of pulse frequency. Auton Neurosci 2006;126-127:72-80.

84. Cunningham JT, Mifflin SW, Gould GG, Frazer A. Induction of c-Fos and DeltaFosB immunoreactivity in rat brain by vagal nerve stimulation. Neuropsychopharmacology 2008;33:18841895.

85. Hammond EJ, Uthman BM, Wilder BJ, et al. Neurochemical effects of vagus nerve stimulation in humans. Brain Res 1992; 583:300-303.

86. Ben-Menachem E, Hamberger A, Hedner T, et al. Effects of vagus nerve stimulation on amino acids and other metabolites in the CSF of patients with partial seizures. Epilepsy Res 1995;20: 221-227.

87. Neese S, Sherill L, Tan A, et al. Vagus nerve stimulation may protect GABAergic neurons following traumatic brain injury in rats: an immunocytochemical study. Brain Res 2007;1128:157163.

88. Marrosu F, Serra A, Maleci A, Puligheddu M, Biggio G, Piga M. Correlation between $\mathrm{GABA}_{\mathrm{A}}$ receptor density and vagus nerve stimulation in individuals with drug-resistant partial epilepsy. Epilepsy Res 2003;55:59-70.

89. Follesa P, Biggio F, Gorini G, et al. Vagus nerve stimulation increases norepinephrine concentration and the gene expression of BDNF and bFGF in the rat brain. Brain Res 2007;1179:28-34.

90. Roosevelt RW, Smith DC, Clough RW, Jensen RA, Browning RA. Increased extracellular concentrations of norepinephrine in cortex and hippocampus following vagus nerve stimulation in the rat. Brain Res 2006;1119:124-132.

91. Hassert DL, Miyashita T, Williams CL. The effects of peripheral vagal nerve stimulation at a memory-modulating intensity on norepinephrine output in the basolateral amygdala. Behav Neurosci 2004 Feb;118:79-88.

92. Borovikova L, Ivanova S, Zhang M, et al. Vagus nerve stimulation attenuates the systemic inflammatory response to endotoxin. Nature 2000;405:458-462.

93. Hosoi T, Okuma Y, Nomura Y. Electrical stimulation of afferent vagus nerve induces IL- $1 \beta$ expression in the brain and activates HPA axis. Am J Physiol Regul Integr Comp Physiol 2000;279: R141-R147.

94. De Herdt V, Dewaele J, Van Aken J, et al. Modulation of seizure threshold by vagus nerve stimulation in an animal model for motor seizures. Epilepsia 2006;47 Suppl 4:300-301 (abstract).

95. Boon P, Vonck K, Van Walleghem P, et al. Programmed and magnet-induced vagus nerve stimulation for refractory epilepsy. J Clin Neurophysiol 2001;18:402-407.

96. Di Lazzaro V, Oliviero A, Pilato F, et al. Effects of vagus nerve stimulation on cortical excitability in epileptic patients. Neurology 2004;62:2310-2312.

97. Naritoku DK, Mikels JA. Vagus nerve stimulation attenuates electrically kindled seizures. Epilepsia 1996;37 Suppl 5:75 (abstract).

98. Fernández-Guardiola A, Martínez A, Valdés-Cruz A, MagdalenoMadrigal VM, Martínez D, Fernández-Mas R. Vagus nerve prolonged stimulation in cats: effects on epileptogenesis (amygdala electrical kindling): behavioural and electrographic changes. Epilepsia 1999;40:822-829.

99. Spencer SS, Guimaraes P, Katz A, Kim J, Spencer D. Morphological patterns of seizures recorded intracranially. Epilepsia 1992;33:537-545.

100. Velasco M, Velasco F, Velasco A, et al. Subacute electrical stimulation of the hippocampus blocks intractable temporal lobe seizures and paroxysmal EEG activities. Epilepsia 2000;41:158 169. 
101. Lian J, Bikson M, Sciortino C, Stacey WC, Durand DM. Local suppression of epileptiform activity by electrical stimulation in rat hippocampus in vitro. J Physiol 2003;547:427-434.

102. Weiss SRB, Li XL, Rosen JB, Li H, Heynen T, Post RM. Quenching: inhibition of development and expression of amygdala kindled seizures with low frequency stimulation. Neuroreport 1995;4:2171-2176.

103. Bragin A, Wilson CL, Engel J Jr. Increased afterdischarge threshold during kindling in epileptic rats. Exp Brain Res 2002;144: 30-37.

104. Velísek L, Velísková J, Stanton PK. Low-frequency stimulation of the kindling focus delays basolateral amygdala kindling in immature rats. Neurosci Lett 2002;326:61-63.
105. Vonck K, Boon P, Achten E, De Reuck J, Caemaert J. Long-term amygdalohippocampal stimulation for refractory temporal lobe epilepsy. Ann Neurol 2002;52:556-565.

106. Boon P, Vonck K, De Herdt V, et al. Deep brain stimulation in patients with refractory temporal lobe epilepsy. Epilepsia 2007; 48:1551-1560.

107. Sun FT, Morrell MJ, Wharen RE Jr. Responsive cortical stimulation for the treatment of epilepsy. Neurotherapeutics 2008;5: $68-74$.

108. Katariwala NM, Bakay RAE, Pennell PB, Olson LD, Henry TR, Epstein CM. Remission of intractable epilepsy following implantation of intracranial electrodes. Neurology 2001;57: $1505-1507$. 